\title{
EFFECTS OF COSMIC RAYS ON HYDROCARBON INTERSTELLAR DUST
}

\author{
M. Godard ${ }^{1,2}$, G. Féraud ${ }^{3}$, M. Chabot ${ }^{4}$, Y. Carpentier ${ }^{3,5}$, T. Pino ${ }^{3}$, \\ R. Brunetto ${ }^{1}$, J. Duprat ${ }^{6}$, C. Engrand ${ }^{6}$, P. Bréchignac ${ }^{3}$, \\ L. d'Hendecourt $^{1}$ and E. Dartois ${ }^{1}$
}

\begin{abstract}
Hydrogenated amorphous carbons, an important component of the interstellar carbonaceous dust, possess infrared spectral signatures (at 3.4, 6.9 and $7.3 \mu \mathrm{m}$ ) that are ubiquitous in the diffuse interstellar medium of galaxies, but not observed in dense clouds. To better understand the role played by cosmic rays in the disappearance of these absorption bands, irradiation experiments of hydrocarbon dust analogues have been performed with different swift ions. The results obtained through the in situ infrared monitoring of the samples during the irradiations allow to infer the dehydrogenation effect of the cosmic ray distribution on the interstellar hydrogenated amorphous carbons. The importance of this interstellar dust destruction by cosmic rays is discussed in comparison to other energetic processes in different interstellar environments.
\end{abstract}

\section{Introduction}

Dust is a major component of the interstellar medium that continuously evolves with the different energetic processes active in such environments (UV photons, cosmic rays, shocks, heating, ...). Observations of the interstellar spectral signatures, together with laboratory simulations of dust processing, allow to get insights into the composition and evolution of the different types of interstellar grains.

\footnotetext{
1 Institut d'Astrophysique Spatiale, Univ. Paris-Sud, Bât. 121, 91405 Orsay, France

2 Astrochemistry Laboratory (Code 691), NASA Goddard Space Flight Center, Building 34, 8800 Greenbelt Road, Greenbelt, MD 20771, USA

3 Institut des Sciences Moléculaires d'Orsay, Univ. Paris-Sud, Bât. 210, 91405 Orsay, France

4 Institut de Physique Nucléaire d'Orsay, Univ. Paris-Sud, Bât. 102, 91405 Orsay, France

5 Laboratoire de Physique des Lasers, Atomes et Molécules, Univ. Lille 1, Bât. P5, 59655 Villeneuve d'Ascq Cedex, France

${ }^{6}$ Centre de Spectrométrie Nucléaire et de Spectrométrie de Masse, Univ. Paris-Sud, Bât. 104, 91405 Orsay, France
} 
One component of the carbonaceous dust is observed through the $3.4 \mu \mathrm{m} \mathrm{ab}-$ sorption band, ubiquitous along sight lines that sample the diffuse interstellar medium of the Milky Way and other galaxies (e.g. Dartois et al. 2004; Pendleton et al. 1994). This feature is attributed to the stretching modes of the $\mathrm{C}-\mathrm{H}$ bonds in methyl $\left(-\mathrm{CH}_{3}\right)$ and methylene $\left(-\mathrm{CH}_{2}-\right)$ groups. The corresponding bending modes (6.85 and $7.25 \mu \mathrm{m})$ are also observed. These interstellar signatures of aliphatic hydrocarbon dust are well reproduced by hydrogenated amorphous carbons (a-C:H or HAC) that can be synthesized in laboratory to produce interstellar analogues. One of the puzzling observations concerning this carbonaceous dust component is the fact that the $3.4 \mu \mathrm{m}$ feature is widely detected in the diffuse interstellar medium, but is not observed in dense clouds. Considering the rapid cycling of dust between these two interstellar phases, it implies an efficient mechanism for the destruction/modification of aliphatic hydrocarbon dust.

As energetic particles are known to dehydrogenate aliphatic materials, exposure of interstellar dust to cosmic rays (CR) could play a role in the disappearance of the $3.4 \mu \mathrm{m}$ feature in dense clouds. A thorough investigation of the CR effects on the carbonaceous dust would help to fully understand its evolution. We have performed experiments of carbonaceous interstellar analogues irradiation by many swift ions. This work (Godard et al. 2011) represents a step further to achieve this goal and extends the work done by Mennella et al. (2003).

\section{Experiments and results}

For these experiments, two kinds of hydrocarbon samples, deposited on KBr substrates, have been used: a-C:H (produced with a hydrocarbon plasma and reproducing the spectral signatures of interstellar ones) (Godard \& Dartois 2010) and soots (aromatic solids that nucleate in a propylene flame) (Pino et al. 2008). These materials have been exposed to many different ions (from hydrogen to iodine) and high energies (between 0.2 and $160 \mathrm{MeV}$ ) at the Tandem accelerator of the Institut de Physique Nucléaire (Orsay, France) and at the Laboratory for Experimental Astrophysics (INAF-Catania, Italy). These swift ions allow to reproduce a wide range of conditions comparable to the distribution of elements and energies of interstellar CR. During the experiments, the induced dehydrogenation and chemical bonding modifications were studied with in situ infrared spectroscopy.

The decreasing optical depth of the aliphatic $\mathrm{C}-\mathrm{H}$ features with the increasing fluence ( $c f$. Fig. 1) has been carefully analyzed with a model describing the mechanism of hydrogen loss in the material (Adel et al. 1989; Marée et al. 1996) and based on the fact that hydrogen leaves the irradiated material in molecular form (Möller \& Scherzer 1987; Wild \& Koidl 1987). We obtained the dehydrogenation parameters for each experiment (dehydrogenation cross section and asymptotic hydrogen content remaining in the irradiated material at high fluence) and their dependance with the electronic stopping power $S_{e}$ (i.e. the energy deposited from the ion to electrons of the irradiated material, per length unit). The values obtained for a-C:H, soots, and other hydrocarbon materials found in the literature 
are quite comparable, pointing out that these parameters do not seem very dependent of the H/C ratio for example. Since $S_{e}$ can be calculated for each ion, these results allow thus to infer the dehydrogenation effect by each cosmic ray of given element and energy.

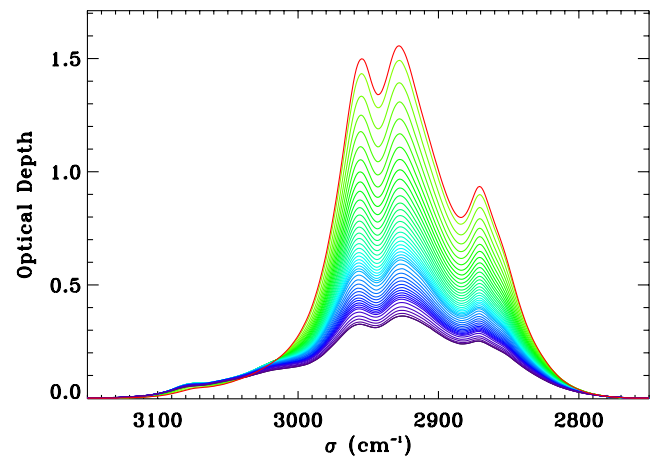

Fig. 1. Example of the decreasing optical depth of the aliphatic C-H stretching IR absorption feature around $2900 \mathrm{~cm}^{-1}(3.4 \mu \mathrm{m})$ during ion irradiation of a-C:H with $85 \mathrm{MeV}$ $\mathrm{Si}^{7+}$, between 0 (red upper line) and $10^{14}$ ions $\mathrm{cm}^{-2}$ (purple lower line). The fluence between two spectra is of the order of few $10^{12}$ ions $\mathrm{cm}^{-2}$.

\section{Effects of cosmic rays inferred from experiments}

With our results, and by adopting an abundance and energy distribution of interstellar cosmic ray flux from the literature (http://galprop.stanford.edu/; Shen et al. 2004), we calculated the summed effect by all cosmic rays (lighter than copper) of different energies (below $10 \mathrm{GeV}$ ) and obtained the evolution of the interstellar aliphatic dust component (and the corresponding spectral signatures) as a function of cosmic ray exposure time. We found that the destruction of aliphatic $\mathrm{C}-\mathrm{H}$ bonds by cosmic rays occurs in characteristic times of a few $10^{8}$ years, a time longer than the characteristic lifetime of an interstellar cloud ( $\sim 10^{7}$ years). Moreover, taking into account the non-zero asymptotic hydrogen content remaining at high fluence shows that, even for longer timescales, the destruction of interstellar a-C:H bonds by cosmic rays only cannot be complete. Even if there are incertitudes on the values of interstellar cosmic ray flux and ionisation rate $^{1}$, a higher $\mathrm{CR}$ flux than the one adopted could not destroy more than $\sim 60 \%$ of this dust component. Thus, cosmic rays destroy interstellar a-C:H, but do not seem efficient enough to explain the disappearance of the $3.4 \mu \mathrm{m}$ band in dense clouds.

This work confirms also that heavy ions play an important role in the destruction, even if they are less abundant. For example, we estimate that iron cosmic rays alone contribute between $5 \%$ and $40 \%$ to the destruction, although they represent only one cosmic ray over 10000 .

These results, concerning the exposure to cosmic rays deduced from experiments, help to better understand the evolution of interstellar a-C:H. They can be compared to the processing of hydrogenated amorphous carbons by UV photons

\footnotetext{
${ }^{1}$ We have checked that the adopted cosmic ray flux was compatible with the observed interstellar ionisation rate of hydrogen by CR.
} 
(Mennella et al. 2001) and H atoms (Mennella 2006) in the different interstellar regions. In diffuse interstellar medium, the formation by atomic hydrogen prevails over the destruction by UV photons. Relatively to other processing, destruction by cosmic rays is negligible in diffuse interstellar medium, but not in dense clouds where UV cannot penetrate and the hydrogen is mostly molecular. However, CR are not efficient enough to completely dehydrogenate the $3.4 \mu \mathrm{m}$ carriers during the cloud lifetime. This interstellar component should thus be destroyed in interfaces between diffuse and dense regions, where photons still penetrate but hydrogen, already in molecular form, cannot compensate this destruction.

\section{References}

Adel, M.E., Amir, O., Kalish, R., \& Feldman, L.C., 1989, J. Appl. Phys., 66, 3248

Dartois, E., Marco, O., Muñoz-Caro, G.M., Brooks, K., Deboffle, D., \& d'Hendecourt, L., 2004, A\&A, 423, 549

Godard, M., \& Dartois, E., 2010, A\&A, 519, A39

Godard, M., Féraud, G., Chabot, M., et al., 2011, A\&A, 529, A146

Marée, C., Vredenberg, A., \& Habraken, F., 1996, Mater. Chem. Phys., 46, 198

Mennella, V., 2006, ApJ, 647, L49

Mennella, V., Baratta, G.A., Esposito, A., Ferini, G., \& Pendleton, Y.J., 2003, ApJ, 587, 727

Mennella, V., Muñoz Caro, G.M., Ruiterkamp, R., et al., 2001, A\&A, 367, 355

Möller, W., \& Scherzer, B.M.U., 1987, Appl. Phys. Lett., 50, 1870

Pendleton, Y.J., Sandford, S.A., Allamandola, L.J., Tielens, A.G.G.M., \& Sellgren, K., 1994, ApJ, 437, 683

Pino, T., Dartois, E., Cao, A.-T., et al., 2008, A\&A, 490, 665

Shen, C.J., Greenberg, J.M., Schutte, W.A., \& van Dishoeck, E.F., 2004, A\&A, 415, 203

Wild, C., \& Koidl, P., 1987, Appl. Phys. Lett., 51, 1506 